\title{
ARTICLE
}

Acute myeloid leukemia

\section{Cytogenetics and gene mutations influence survival in older patients with acute myeloid leukemia treated with azacitidine or conventional care}

\author{
Hartmut Döhner ${ }^{1} \cdot$ Anna Dolnik ${ }^{1} \cdot$ Lin Tang $^{2} \cdot$ John F. Seymour $^{3} \cdot$ Mark D. Minden $^{4} \cdot$ Richard M. Stone $^{5}$. \\ Teresa Bernal del Castillo ${ }^{6} \cdot$ Haifa Kathrin Al-Ali ${ }^{7}$. Valeria Santini ${ }^{8} \cdot{\text { Paresh Vyas } \mathbb{1}^{9} \cdot \text { C. L. Beach }}^{2} \cdot$ Kyle J. MacBeth $^{2}$. \\ Barry S. Skikne ${ }^{2} \cdot$ Steve Songer $^{2} \cdot$ Nora $\mathrm{Tu}^{2} \cdot$ Lars Bullinger $^{1,10} \cdot$ Hervé Dombret $^{11}$
}

Received: 28 May 2018 / Revised: 24 July 2018 / Accepted: 6 August 2018 / Published online: 1 October 2018

(c) The Author(s) 2018. This article is published with open access

\begin{abstract}
Older patients with newly diagnosed acute myeloid leukemia (AML) in the phase 3 AZA-AML-001 study were evaluated at entry for cytogenetic abnormalities, and a subgroup of patients was assessed for gene mutations. Patients received azacitidine $75 \mathrm{mg} / \mathrm{m}^{2} /$ day $\mathrm{x} 7$ days $(n=240)$ or conventional care regimens $(\mathrm{CCR} ; n=245)$ : intensive chemotherapy, low-dose cytarabine, or best supportive care only. Overall survival (OS) was assessed for patients with common (occurring in $\geq 10 \%$ of patients) cytogenetic abnormalities and karyotypes, and for patients with recurring gene mutations. There was a significant OS improvement with azacitidine vs CCR for patients with European LeukemiaNet-defined Adverse karyotype (HR 0.71 [95\%CI 0.51-0.99]; $P=0.046)$. Azacitidine-treated patients with $-5 / 5 \mathrm{q}-,-7 / 7 \mathrm{q}-$, or $17 \mathrm{p}$ abnormalities, or with monosomal or complex karyotypes, had a 31-46\% reduced risk of death vs CCR. The most frequent gene mutations were DNMT3A (27\%), TET2 (25\%), IDH2 (23\% [R140, 15\%; R172, 8\%]), and TP53 (21\%). Compared with wild-type, OS was significantly reduced among CCR-treated patients with TP53 or NRAS mutations and azacitidine-treated patients with FLT3 or TET2 mutations. Azacitidine may be a preferred treatment for older patients with AML with Adverse-risk cytogenetics, particularly those with chromosome 5, 7, and/or 17 abnormalities and complex or monosomal karyotypes. The influence of gene mutations in azacitidine-treated patients warrants further study.
\end{abstract}

These authors contributed equally: Lars Bullinger, Hervé Dombret

Co-senior authors: Lars Bullinger, Hervé Dombret

Electronic supplementary material The online version of this article (https://doi.org/10.1038/s41375-018-0257-z) contains supplementary material, which is available to authorized users.

$\triangle$ Hartmut Döhner

hartmut.doehner@uniklinik-ulm.de

1 Ulm University Hospital, Ulm, Germany

2 Celgene Corporation, Summit, NJ, United States

3 Peter MacCallum Cancer Centre, Royal Melbourne Hospital, Melbourne, and University of Melbourne, Parkville, Australia

4 University of Toronto, Toronto, ON, Canada

5 Dana-Farber Cancer Institute, Boston, MA, United States

\section{Introduction}

Acute myeloid leukemia (AML) is associated with a range of recurring cytogenetic abnormalities and gene mutations [1-4]. While the prognostic importance of cytogenetics in AML has been established for decades [5], due to recent advances in next-generation sequencing and greater availability of myeloid-focused gene panels, some genes frequently mutated in AML have been identified that are predictive of treatment response $[2,3,6]$. Molecular genetic

6 Hospital Central de Asturias, Oviedo, Spain

7 Universitätsklinikum Halle (Saale), Halle, Germany

8 AOU Careggi, University of Florence, Florence, Italy

9 University of Oxford, Oxford, United Kingdom

10 Charité University Medicine, Berlin, Germany

11 Hôpital Saint Louis, Institut Universitaire d'Hématologie, Université Paris Diderot, Paris, France 
data are increasingly being used to inform disease classification, risk stratification, and clinical care of patients $[4,7]$. Two provisional entities, AML with mutated RUNXI and AML with $B C R-A B L 1$, have been included in the 2016 update of the World Health Organization (WHO) classification of myeloid neoplasms and acute leukemia [7]. Mutational testing for NPM1, CEBPA, and FLT3 is advised in the 2010 European LeukemiaNet (ELN) recommendations for AML [1], and the 2017 update to the ELN recommendations lists three additional genes-RUNX1, ASXL1, and TP53-that can inform risk stratification, mainly based on experience with intensive chemotherapy (IC) in relatively younger patients [4]. Patterns of comutations have also been identified that have distinct prognostic implications in AML [3].

In the randomized, phase 3 AZA-AML-001 study of older patients with newly diagnosed AML (NCT01074047), azacitidine prolonged median overall survival (OS) vs conventional care regimens (CCR) (10.4 vs 6.5 months, respectively; $P=0.101$ ), with 1 -year survival rates of $46.5 \%$ vs $34.2 \%$, respectively [8]. A prospective subanalysis from the study showed a significantly prolonged OS of 3.2 months with azacitidine compared with CCR (hazard ratio [HR] 0.68, 95\% confidence interval [95\%CI] $0.50,0.94)$ in the subgroup of patients with poor-risk cytogenetics, as defined by National Comprehensive Cancer Network (NCCN) 2009 criteria [8, 9]. That analysis did not investigate outcomes associated with specific cytogenetic abnormalities.

Here we evaluate survival outcomes in patient subgroups from the AZA-AML-001 study, defined by 2010 ELN cytogenetic risk classification and by the presence of specific cytogenetic abnormalities or gene mutations at baseline. Pretreatment cytogenetic risk classification was an entry criterion and cytogenetic data were available for almost all patients. A subpopulation of patients in the AZAAML-001 study with available baseline bone marrow samples for molecular analyses consented to participate in exploratory analyses, to evaluate the frequency of recurring gene mutations at entry and relationships between pretreatment mutational status and OS.

\section{Methods}

\section{Study design}

Full study design, patient eligibility criteria, and response endpoints are described in detail elsewhere [8]. Briefly, patients aged $\geq 65$ years with newly diagnosed AML, $>30 \%$ bone marrow blasts, Eastern Cooperative Oncology Group performance status (ECOG PS) scores $\leq 2$, white blood cell counts $\leq 15 \times 10^{9} / \mathrm{L}$, and intermediate- or poor-risk cytogenetics per 2009 NCCN guidelines for AML [9], were eligible to participate. This study was approved by all relevant institutional review boards or independent ethics committees and was conducted according to the Declaration of Helsinki. All patients provided written informed consent.

Patients were preselected to 1 of 3 CCR: IC (cytarabine $100-200 \mathrm{mg} / \mathrm{m}^{2}$ IV for 7 days + anthracycline IV for 3 days induction), low-dose cytarabine (LDAC; $20 \mathrm{mg}$ SC BID for 10 days per 28-day cycle), or best supportive care (BSC) only. After preselection, patients were randomized 1:1 to azacitidine $\left(75 \mathrm{mg} / \mathrm{m}^{2} /\right.$ day SC for 7 consecutive days per 28-day cycle) or to CCR; those randomized to CCR received their preselected regimen. All patients could receive $\mathrm{BSC}$ as needed.

\section{Cytogenetic analyses}

Karyotypes from pretreatment bone marrow samples were determined locally and karyograms were prepared and sent for central review by an independent cytogeneticist (Anne Hagemeijer, MD). For these analyses, cytogenetic risk status was determined according to modified 2010 ELN recommendations [1], but molecular markers were not considered for risk-group assessments, as they were not available for all patients. Patient subgroups were identified based on ELN-defined cytogenetic risk classifications: Intermediate-I (normal karyotype), Intermediate-II (comprising all abnormalities not classified as Favorable or Adverse), and Adverse (Fig 1). OS outcomes associated with specific cytogenetic abnormalities observed in $\geq 10 \%$ of patients, and with complex or monosomal karyotypes, were also evaluated. Complex karyotype was defined as three or more cytogenetic abnormalities in the absence of a WHOdesignated recurring translocation or inversion; i.e., $\mathrm{t}(8 ; 21)$ $(\mathrm{q} 22 ; \mathrm{q} 22.1) ; \mathrm{t}(9 ; 11)(\mathrm{p} 21.3 ; \mathrm{q} 23.3) ; \operatorname{inv}(3)(\mathrm{q} 21.3 \mathrm{q} 26.2)$ or $\mathrm{t}(3 ; 3)(\mathrm{q} 21.3 ; \mathrm{q} 26.2) ; \quad \mathrm{t}(6 ; 9)(\mathrm{p} 23 ; \mathrm{q} 34.1) ; \quad \mathrm{t}(\mathrm{v} ; 11 ; \mathrm{q} 23.3)$. Monosomal karyotype was defined as the presence of a single monosomy (excluding loss of one $\mathrm{X}$ or $\mathrm{Y}$ chromosome) in association with one or more additional monosomy or structural chromosomal abnormality. Patients with multiple lesions may have been assigned to and evaluated in more than one category. Because the majority (64\%) of all patients in AZA-AML-001 were preselected to receive LDAC before randomization, OS was also compared among LDAC-preselected patients who received azacitidine vs those who received LDAC. The small number of patients preselected to BSC or IC precluded statistical comparisons between cytogenetic abnormality subgroups.

\section{Mutational analyses}

DNA was isolated from pretreatment bone marrow mononuclear cells and targeted sequencing of 39 genes was 


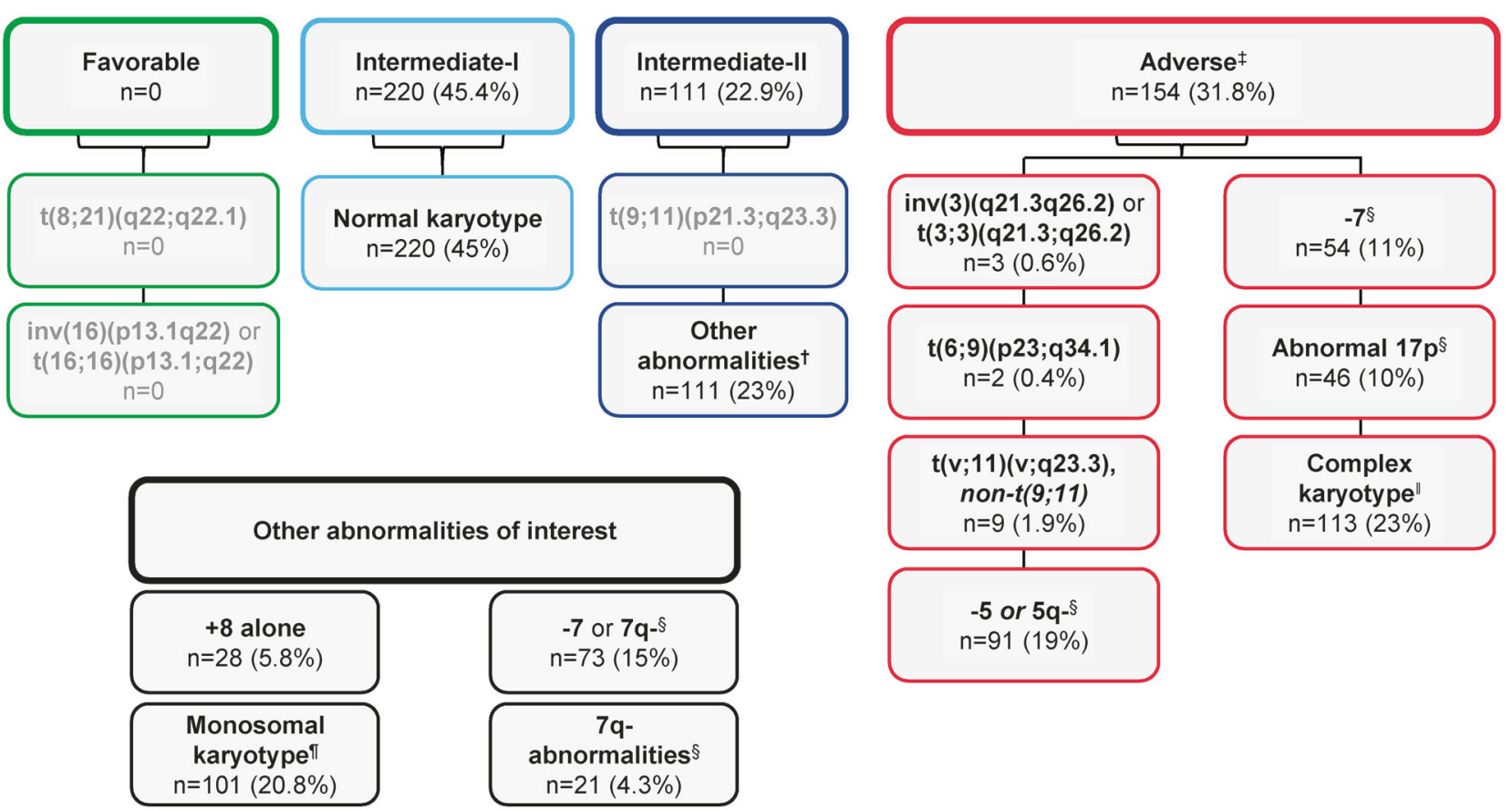

\begin{abstract}
* Not considering NPM1, CEBPA, or FLT3 mutations, as these data were not available for the entire cohort; ${ }^{\dagger}$ Includes patients with cytogenetic abnormalities not included in the Favorable or Adverse categories;

‡Patients with adverse cytogenetics may have been included in more than 1 subgroup; ${ }^{\S}$ Includes all cases, irrespective of whether occurring alone or with other chromosomal abnormalities

" 3 or more abnormalities in the absence of a WHO-designated recurring translocation or inversion; ie, $\mathrm{t}(8 ; 21)(\mathrm{q} 22 ; \mathrm{q} 22.1)$;

$\mathrm{t}(9 ; 11)(\mathrm{p} 21.3 ; \mathrm{q} 23.3)$; inv(3)(q21.3q26.2) or $\mathrm{t}(3 ; 3)(\mathrm{q} 21.3 ; \mathrm{q} 26.2) ; \mathrm{t}(6 ; 9)(\mathrm{p} 23 ; \mathrm{q} 34.1) ; \mathrm{t}(\mathrm{v} ; 11(\mathrm{v} ; \mathrm{q} 23.3)$

" Presence of a single monosomy (excluding loss of $X$ or $Y$ ) in association with $\geq 1$ additional monosomy or structural chromosomal abnormality (excluding core-binding factor AML)
\end{abstract}

Fig. 1 Patient subgroups according to modified 2010 ELN criteria* and frequency of specific chromosomal abnormalities or karyotypes

performed with Haloplex target enrichment (Agilent) on Illumina HiSeq 2500 using $2 \times 100 \mathrm{bp}$ read lengths. Burrows-Wheeler Aligner (BWA)-MEM alignment to genome (hg19) and VarScan v2.3.9 software (Genome Institute at Washington University), a platform-independent tool, were used to detect variants. Target regions varied by gene from all exons to hotspots. Variant annotation filtering included functionally deleterious variants (SnpEff v4.0) functional prediction as non-synonymous SNV/INDEL in exons, splicing regions, and stop sites. Pindel (v0.2.5b5) was used to detect deletions, inversions, small insertions, and tandem duplicates with the parameter setting at a minimum coverage of 10 with a minimum of 5 supporting reads. Heterozygous variant allele frequency (VAF) detection threshold was $3 \%$. There was a total of 312 variants in the combined Pindel and VarScan results. FLT3 tyrosine kinase domain (TKD) mutations were determined by nextgeneration sequencing, and internal tandem duplications (ITD) were determined by capillary electrophoresis sizing of polymerase chain reaction (PCR) amplicons from exons 14 and 15 (binary call; no allelic ratio data were available).

\section{Statistical methods}

Median OS and 1-year survival rates are estimated using Kaplan-Meier methods. OS comparisons according to 2010 ELN cytogenetic risk status, specific cytogenetic abnormalities, and karyotype are made using a weighted log-rank test. HRs and 95\%CIs are from an unstratified Cox proportional hazards model; $P$ values are from weighted logrank tests (not adjusted for multiplicity of testing).

Within each treatment arm (azacitidine or CCR), OS was compared between patients with specific gene mutations vs those with corresponding wild-type genes. Additionally, OS comparisons were made between the azacitidine and CCR arms for patients with gene mutations detected in $\geq 5$ patients. OS comparisons by gene mutational status are made using a log-rank test stratified by baseline ECOG PS score ( $0-1$ vs 2$)$ and NCCN cytogenetic risk (intermediate vs poor).

The influence of VAFs of gene mutations found to significantly influence OS in univariate analyses was investigated in two Cox proportional hazards models [10]. 
Relative hazards were simulated for VAF from the Cox proportional hazards models and plotted against the VAF to visualize the effect of VAF on OS. One model evaluated relative OS hazards by mutant VAF as a continuous variable vs OS in patients with wild-type corresponding genes $(\mathrm{VAF}=0)$, with treatment (azacitidine, CCR) as strata in the model. The second model investigated the relative OS hazards by baseline mutation VAFs vs wild-type genes within the azacitidine and CCR arms. When multiple loci were mutated within a gene, the mutation with the highest VAF was used in the model.

\section{Results}

\section{Patients}

The intention-to-treat population in AZA-AML-001 included 488 patients (azacitidine, $n=241$; CCR, $n=247$ ) [8]. Of them, centrally reviewed cytogenetic data were available for 485 patients (99.4\%; azacitidine, $n=240$; CCR, $n=$ 245 , including IC $[n=44]$, LDAC $[n=158]$, and BSC only $[n=45])$. In all, 220 patients $(45.4 \%)$ had ELN-defined Intermediate-I risk (i.e., normal) karyotype (azacitidine, $n=114$; CCR, $n=106)$, 111 patients (22.9\%) had an Intermediate-II risk karyotype (azacitidine, $n=53$; CCR, $n=58)$, and 154 patients $(31.8 \%)$ had an Adverse risk karyotype (azacitidine, $n=73$; CCR, $n=81$ ) (Fig. 1). Baseline characteristics of the cytogenetic analysis population were essentially unchanged from those of all patients in the AZA-AML-001 study [8].

The "biomarker cohort" comprised 156 patients who were assessed at study entry for presence of gene mutations (azacitidine, $n=83$; CCR, $n=73$ ). Baseline characteristics were generally similar between azacitidinetreated and CCR-treated patients (Supplementary Table 1). Prior history of myelodysplastic syndromes (MDS) was somewhat more common in azacitidine-treated patients ( $23 \%$ vs $14 \%$ of CCR patients) and CCR-treated patients were proportionally more likely to have ELN-defined Adverse risk cytogenetics (44\% vs $33 \%$ ).

\section{Cytogenetic analyses}

Median OS was comparable between azacitidine and CCR among patients with Intermediate-I risk (14.1 vs 10.1 months, respectively; HR 0.83 [95\%CI 0.60, 1.1]; $P=$ $0.44)$ or Intermediate-II risk (8.9 vs 9.6 months; HR 1.19 [95\%CI $0.79,1.8] ; P=0.78$ ) cytogenetics (Fig. 2). Estimated 1-year survival rates in the Intermediate-I risk group were $60.1 \%$ with azacitidine and $45.5 \%$ with CCR, and in the Intermediate-II group were $41.5 \%$ and $42.1 \%$, respectively. There was a significant difference in median OS in favor of azacitidine among patients with Adverse risk karyotypes (5.3 vs 2.9 months with CCR; HR 0.71 [95\%CI $0.51,0.99$ ]; $P=0.046$ ), with estimated 1 -year survival rates of $29.1 \%$ vs $14.7 \%$ for patients treated with azacitidine and CCR, respectively.

The LDAC preselection group included 154 patients treated with azacitidine and 158 patients treated with LDAC. Median OS with azacitidine and LDAC was 13.3 vs 12.5 months, respectively (HR 1.1 [95\%CI $0.75,1.6]$ ), among patients with Intermediate-I risk cytogenetics, 10.7 vs 5.6 months $(0.93[0.56,1.5])$ among patients with Intermediate-II risk cytogenetics, and 5.9 vs 4.3 months $(0.73[0.47,1.1])$ for those with Adverse risk karyotypes.

Among all patients, those with complex karyotypes $(n=$ $113 ; 23 \%$ ), those treated with azacitidine had a statistically significant improvement in OS compared with those who received CCR (median 4.8 months vs 2.8 months, respectively; HR 0.64 [95\%CI 0.43, 0.94]; $P=0.037$ ) (Fig. 3), with an estimated $15 \%$ more azacitidine-treated patients alive at 1 year $(22.8 \%$ vs $7.9 \%)$. There was also a trend for improvement in median OS with azacitidine for patients with monosomal karyotypes $(n=101,21 \%)(5.0$ vs 2.8 months with CCR; HR 0.65 [95\%CI 0.42, 1.01]; $P=$ 0.055 ), with estimated 1-year survival rates of $19.6 \%$ vs $7.8 \%$, respectively. Within the LDAC preselection group, median OS among patients with complex karyotypes was 5.3 months with azacitidine vs 2.9 months with LDAC (HR 0.61 [95\%CI $0.36,1.0]$ ), and for patients with monosomal karyotypes was 5.9 months vs 2.9 months, respectively (HR $0.66[0.37,1.2]$ ).

Specific cytogenetic abnormalities observed in $\geq 10 \%$ of patients occurred in chromosomes 5 (19\%), 7 (15\%), and 17p (10\%) (Fig. 1). Compared with CCR, treatment with azacitidine was associated with significantly longer median OS for patients with complex karyotypes or -7/7qabnormalities, and showed a trend for improved survival in patients with monosomal karyotypes or abnormalities in chromosomes 5 and 17 (Fig. 3). Median OS in the CCR arm was less than 3 months for patients in each of these subgroups.

\section{Mutational analyses}

Molecular abnormalities were detected in 33 of the 39 sequenced genes (Fig. 4) and in 153 (98.1\%) of the 156 patients in the biomarker population. The most frequently mutated genes were DNMT3A (27\%), TET2 (25\%), IDH2 (23\% [-R140, 15\%; -R172, 8\%]), TP53 (21\%), RUNX1 (18\%), NPM1 (16\%), NRAS (12\%), FLT3 (12\% [-ITD, 10\%; -TKD, 5\%]), ASXL1 (11\%), and STAG2 (10\%). No mutations were found in BRAF, DNMT1, DNMT3B, FAM5C, HNRNPK, or PTEN genes. No patient with a TP53 mutation had a co-occurring NPM1 or RUNX1 mutation 
Intermediate I

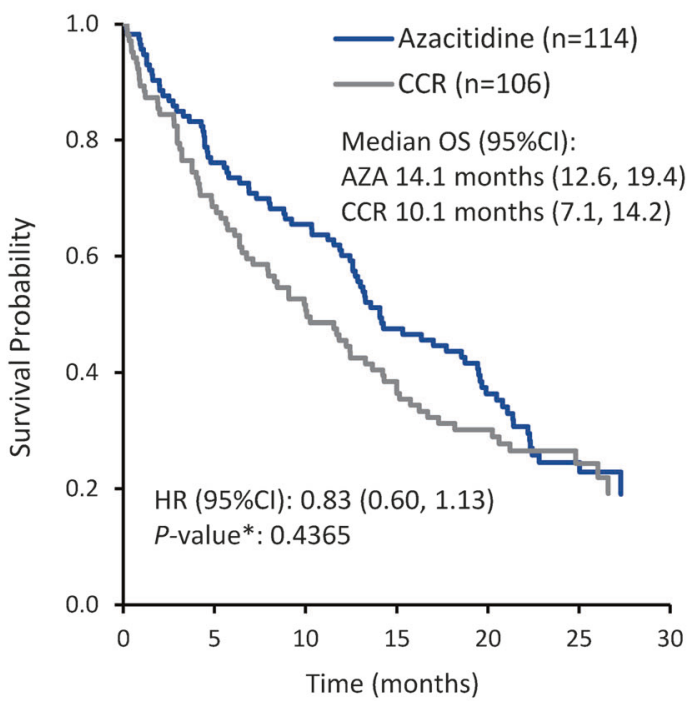

Intermediate II

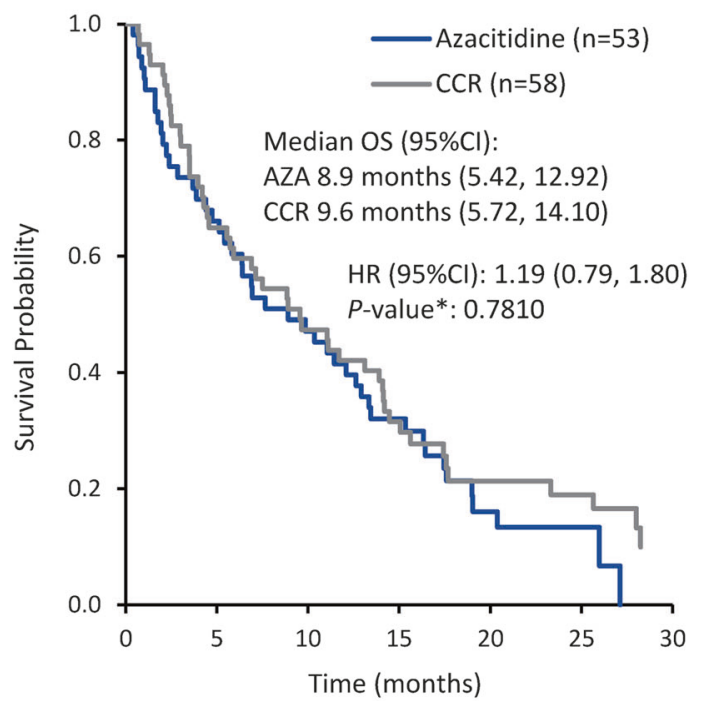

Adverse

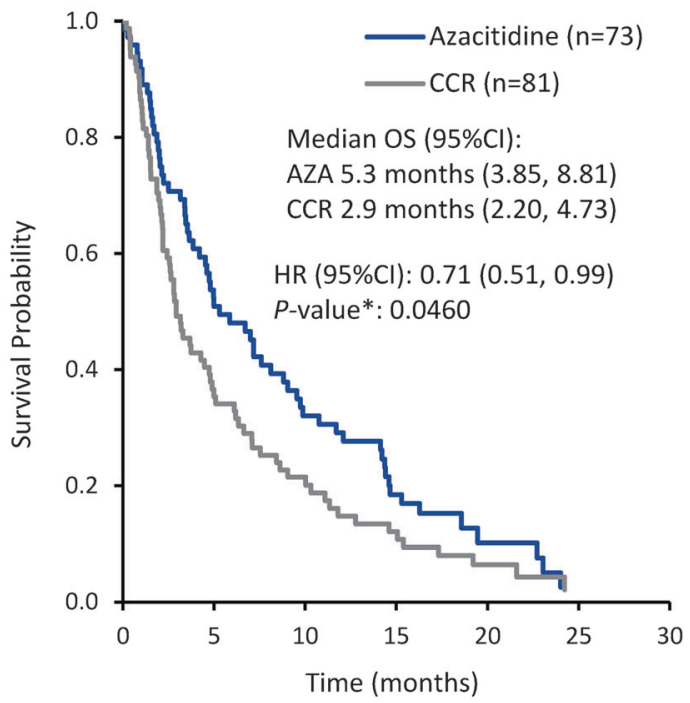

* $P$ values from weighted log-rank test

Fig. 2 Overall survival associated with cytogenetic risk groups (per modified 2010 ELN criteria)

( $P=0.002$ and $P=0.001$, respectively), and $50 \%$ of patients with an FLT3 mutation also had an NPM1 mutation $(P<0.001)$ (Supplementary Figure 1). No patient with an NPM1 mutation had a co-occurring RUNX1 or ASXL1 mutation ( $P=0.004$ and $P=0.043$, respectively). Only 3 patients had a $C E B P A$ mutation and all were monoallelic.

Median OS did not differ significantly in the azacitidine and CCR arms among patients with 1, 2, or $\geq 3$ gene mutations at study entry. Within treatment arms, four gene mutations were significantly correlated with OS compared with wild-type genes: TP53, NRAS, FLT3 (including both -ITD and -TKD), and TET2 (Table 1). In the CCR arm, median OS was significantly reduced for patients with TP53 mutations $(n=17)$ compared with wild-type $(n=56)(2.4$ vs 12.5 months, respectively; $P=0.026)$ and for patients with mutant NRAS $(n=8)$ vs wild-type NRAS $(n=65)(4.3$ vs 10.3 months, respectively; $P=0.020$ ) (Fig. 5). Within the azacitidine arm, median OS was not significantly different between patients with $(n=15)$ or without $(n=68)$ TP53 mutations (7.2 vs 12.0 months, respectively; $P=$ $0.40)$ or between patients with mutant $(n=10)$ or wild-type ( $n=73$ ) NRAS (11.8 vs 8.9 months; $P=0.95)$. However, median OS in the azacitidine arm was reduced in patients with mutant FLT3 $(n=9)$ vs wild-type FLT3 $(n=74)(5.4$ vs 12.0 months, respectively; $P=0.017)$. Despite similar median OS, there was a statistically significant difference $(P=0.005)$ in OS within the azacitidine arm for patients with TET2 mutations $(n=22)$ vs those with wild-type TET2 

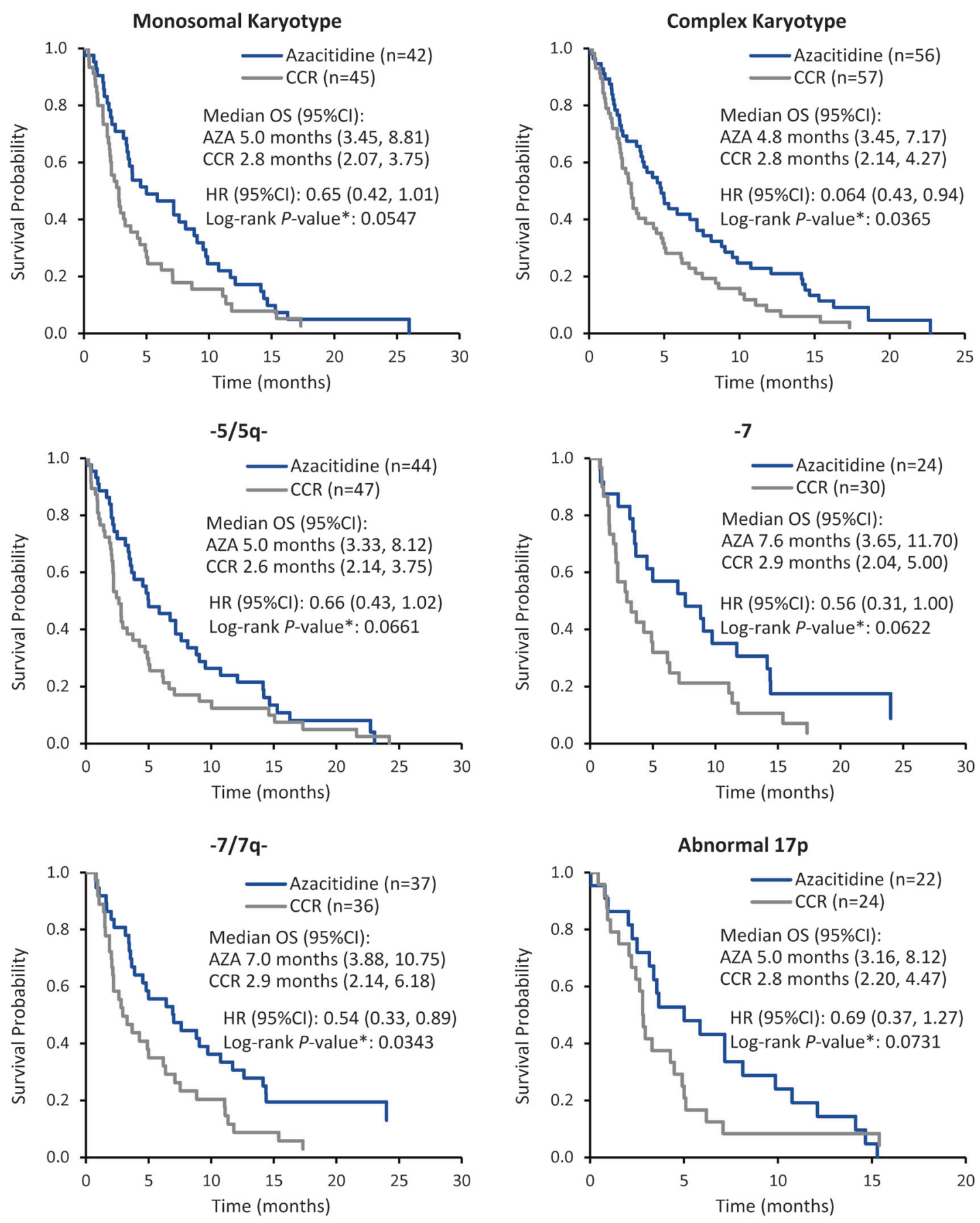

* $P$ values from weighted log-rank test

Fig. 3 Overall survival associated with monosomal and complex karyotypes and with specific cytogenetic abnormalities occurring in $\geq 10 \%$ of patients

( $n=61)$ due to separation of the survival curves after the median (Fig. 5). Median OS within the azacitidine and CCR treatment arms was comparable for patients with or without mutations in any of the genes known to influence DNA methylation (i.e., DNMT3A, IDH1, IDH2, TET1, and TET2). There were no statistically significant survival differences within either treatment arm for patients with known or provisional class-defining lesions (e.g., RUNX1, NPM1) (Table 1), or any other gene mutation evaluated compared with OS in patients with corresponding wild-type genes (Supplementary Figure 2).

Survival comparisons between the azacitidine and CCR arms indicated that patients with mutant TP53 or $N R A S$ treated with azacitidine had nominally better median 


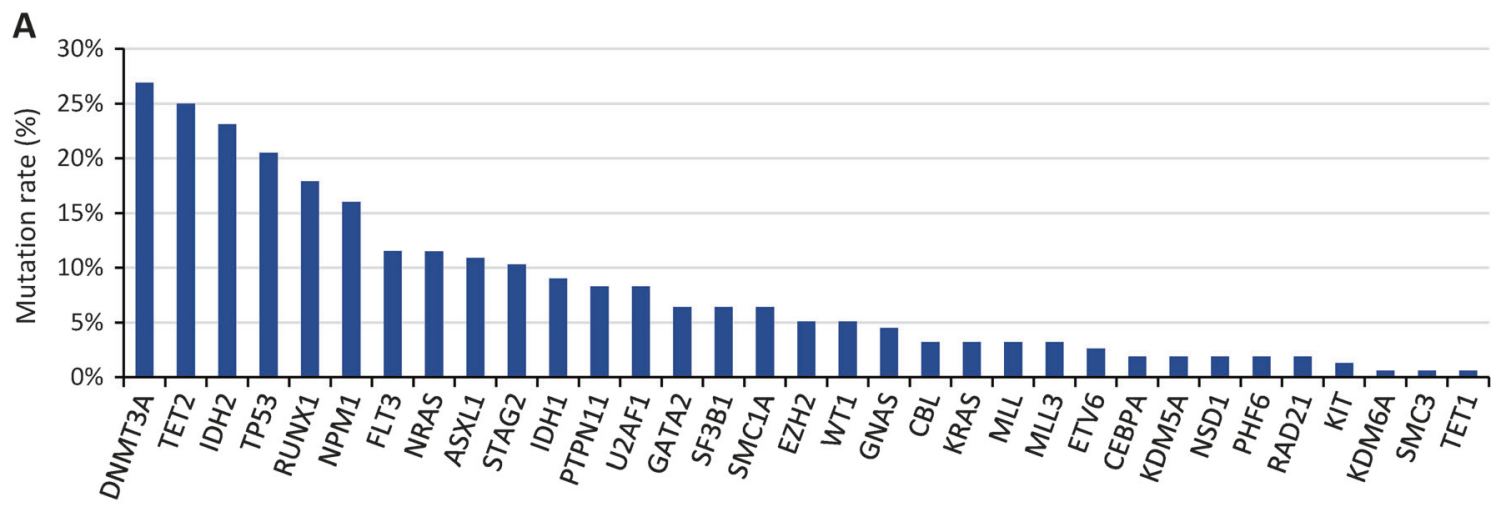

B

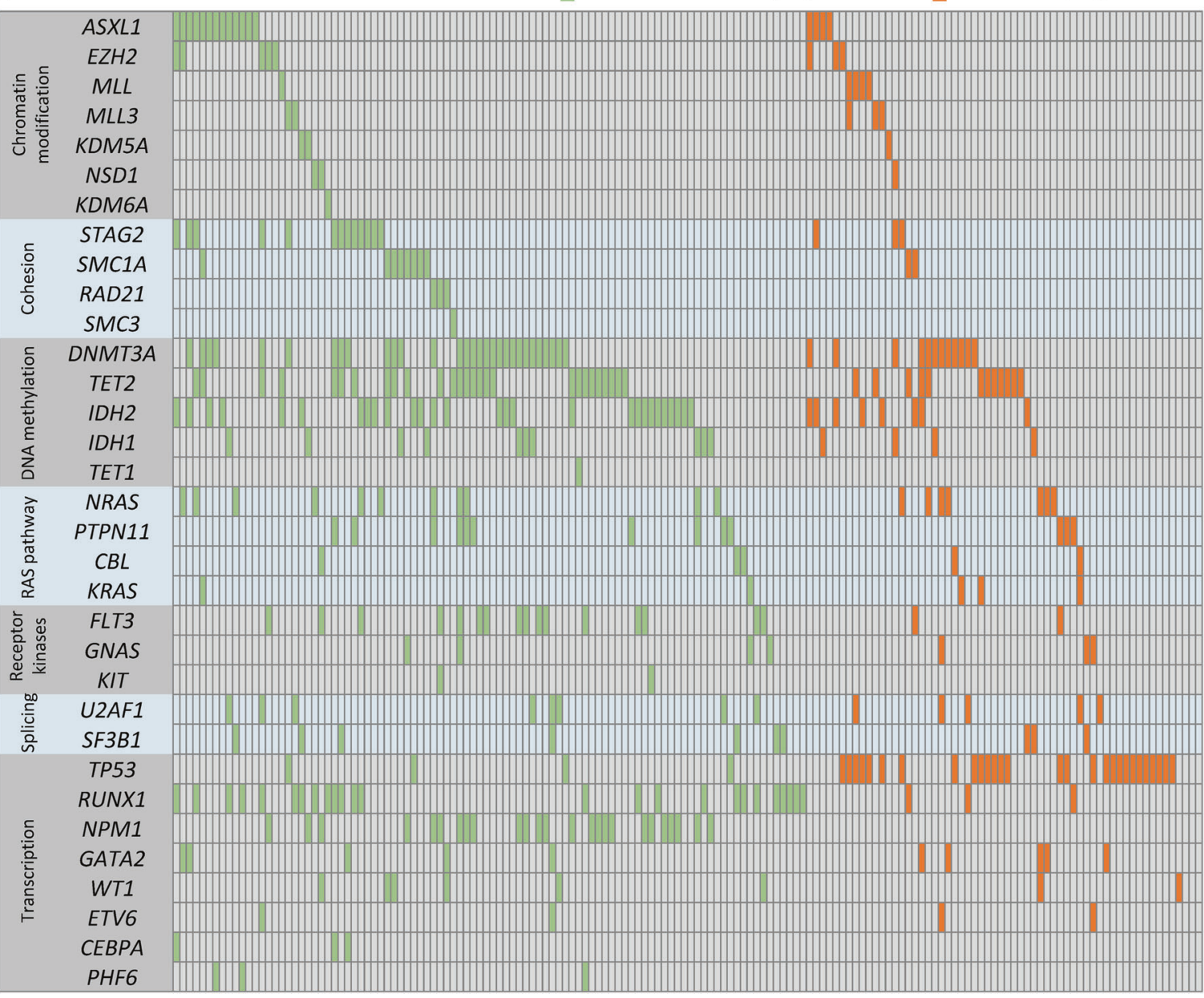

Fig. 4 a Proportions of patients with specific gene mutations. b Oncoplot showing gene mutations in individual patients with intermediate-I/II risk (green) or poor-risk (orange) cytogenetics

OS than their counterparts in the CCR arm: median OS was 7.2 vs 2.4 months, respectively, for patients with mutant TP53, and 11.8 vs 4.3 months for those with mutant NRAS (Table 2). Conversely, azacitidine-treated patients with TET2 mutations had worse OS outcomes than CCR-treated patients with the mutation (median OS 9.6 vs 11.1 months, respectively; $P=0.036$ ). Median OS was similar between treatment groups for patients with a mutation in any of the DNA methylation genes.

The influence of VAFs of mutant TP53, NRAS, FLT3TKD, and TET2 at baseline on OS $v s$. wild-type genes in the Cox model stratified by treatment showed a significant increase in relative hazards on OS with increasing mutant TP53 $(P<0.0001)$ and TET2 $(P=0.042) \quad$ VAFs 
Table 1 Median OS within treatment arms (mutant vs wild type) for the most frequently ( $\geq 10 \%$ of patients) mutated genes and genes involved in DNA methylation

\begin{tabular}{|c|c|c|c|c|c|c|}
\hline \multirow[t]{2}{*}{ Mutated gene ${ }^{a}$} & \multicolumn{3}{|c|}{ AZA } & \multicolumn{3}{|c|}{ CCR } \\
\hline & $\begin{array}{l}\text { WT Median OS, } \\
\text { months }(95 \% \mathrm{CI})\end{array}$ & $\begin{array}{l}\text { MUT Median OS, } \\
\text { months }(95 \% \mathrm{CI})\end{array}$ & $\begin{array}{l}\text { Stratified } \\
P \text { value }\end{array}$ & $\begin{array}{l}\text { WT Median OS, } \\
\text { months }(95 \% \mathrm{CI})\end{array}$ & $\begin{array}{l}\text { MUT Median OS, } \\
\text { months }(95 \% \mathrm{CI})\end{array}$ & $\begin{array}{l}\text { Stratified } \\
P \text { value }\end{array}$ \\
\hline TP53 & $12.0(7.0,16.3)$ & $7.2(3.9,18.6)$ & 0.404 & $12.5(9.6,17.6)$ & $2.4(1.5,7.1)$ & 0.026 \\
\hline$N R A S$ & $8.9(5.8,14.3)$ & $11.8(7.7, \mathrm{NR})$ & 0.946 & $10.3(6.4,15.1)$ & $4.3(2.3, \mathrm{NR})$ & 0.020 \\
\hline$F L T 3^{\mathrm{a}}$ & $12.0(7.6,16.3)$ & $5.4(4.5, \mathrm{NR})$ & 0.017 & $9.6(5.1,14.6)$ & $6.4(3.8, \mathrm{NR})$ & 0.272 \\
\hline TET2 & $9.5(6.9,18.7)$ & $9.6(4.5,13.5)$ & 0.005 & $7.1(5.6,14.2)$ & $11.1(2.8, \mathrm{NR})$ & 0.445 \\
\hline$I D H 2$ & $9.2(7.0,13.3)$ & $12.6(4.4, \mathrm{NR})$ & 0.602 & $6.8(4.9,14.1)$ & $12.5(5.6, \mathrm{NR})$ & 0.466 \\
\hline DNMT3A & $8.2(4.8,14.3)$ & $12.6(7.0,20.8)$ & 0.413 & $8.6(5.1,14.3)$ & $10.3(3.8, \mathrm{NR})$ & 0.597 \\
\hline$R U N X 1$ & $8.3(5.1,13.3)$ & $13.5(8.8, \mathrm{NR})$ & 0.718 & $6.1(3.8,11.7)$ & $15.8(12.5, \mathrm{NR})$ & 0.084 \\
\hline NPM1 & $10.3(7.2,14.3)$ & $7.3(4.5, \mathrm{NR})$ & 0.260 & $9.6(5.1,14.2)$ & $6.4(3.8, \mathrm{NR})$ & 0.698 \\
\hline$A S X L 1$ & $8.9(6.9,13.2)$ & $18.7(4.8, \mathrm{NR})$ & 0.229 & $7.1(5.1,14.1)$ & $14.6(10.0, \mathrm{NR})$ & 0.498 \\
\hline STAG2 & $8.8(5.8,13.2)$ & $19.5(11.9, \mathrm{NR})$ & 0.469 & $8.6(5.6,14.2)$ & $11.1(5.1, \mathrm{NR})$ & 0.395 \\
\hline $\begin{array}{l}\text { Any DNA } \\
\text { methylation gene }\end{array}$ & $8.8(5.4,18.7)$ & $11.1(5.8,15.3)$ & 0.357 & $6.7(4.9,14.2)$ & $12.5(4.3,17.6)$ & 0.299 \\
\hline
\end{tabular}

${ }^{\mathrm{a}}$ FLT3-ITD and FLT3-TKD

bIncludes IDH1, IDH2, DNMT3A, TET1, and TET2

MUT mutant gene(s), NR not reached, $O S$ overall survival, $W T$ wild-type gene(s)

(Supplementary Figure 3). In individual treatment arms, the influence of increased TP53 VAF on OS vs wild-type was both negative and significant in the azacitidine and CCR arms but was much stronger in the CCR arm $(P<0.0001$ vs $P=0.058$ in the CCR vs azacitidine arms, respectively) with higher relative hazard on OS in the CCR arm at comparable VAF levels. There was a significant correlation between increased mutant TET2 VAF and OS hazard in the azacitidine arm $(P=0.0091)$ but no VAF influence in the CCR arm $(P=0.97)$ (Supplementary Figure 4$)$.

\section{Discussion}

Prognosis is dismal for older patients with AML and Adverse-risk cytogenetics, including those with complex or monosomal karyotypes. Approximately one-third of patients in AZA-AML-001 had an Adverse karyotype; median OS among azacitidine-treated patients was almost double that of patients treated with CCR. Similarly, azacitidine-treated patients with monosomal or complex karyotypes had 35 and $36 \%$ reduced risks of death, respectively, compared with similar patients who received CCR.

Deletions of part or all of chromosomes 5, 7, or 17 occur in $5-10 \%$ of all patients with AML, are often associated with complex and monosomal karyotypes, and carry a poor prognosis [11-13]. They are more common in older patients and were the most frequent cytogenetic abnormalities in the AZA-AML-001 study population, occurring in 10-19\% of patients. These chromosomal defects are frequently associated with multilineage dysplasia in bone marrow, poor response to chemotherapy, and high relapse rate [14]. In the current analyses, median OS was approximately doubled in azacitidine-treated patients with chromosome 5 , 7 , or 17 abnormalities compared with similar patients who received CCR. Similar to reporting in MDS [15], patients with AML with chromosome 7 abnormalities fared particularly well with azacitidine, with a median OS improvement of 4.4 ( \pm 0.3) months compared with CCR. Analogous effects with azacitidine for treatment of AML and MDS would not be unexpected, as chromosome 5, 7, and 17 defects are diagnostic features of AML with myelodysplasia-related changes (AML-MRC) [4, 16]. The majority of all patients in AZA-AML-001 (54\%) were identified as having AMLMRC upon central cytogenetic review [14]. Better outcomes with azacitidine in patients with these specific cytogenetic abnormalities in the current analysis are consistent with improved survival reported for all azacitidinetreated patients with AML-MRC in this study, who showed a median OS prolonged by 4.0 months compared with CCR (8.9 vs 4.9 months; HR 0.74 [95\%CI 0.57, 0.97]) [14].

The genomic landscape differs between younger and older patients with AML [2]. Mutational frequencies in the AZA-AML-001 "biomarker" population were as might be expected for older patients [17-20]. Mutations in genes encoding epigenetic modifiers, such as DNMT3A, TET2, and $I D H 2$, are more common in older patients and are usually acquired early in the evolution of the disease, often present in the founding clone. Similarly, mutations that are acquired later (e.g., NPM1 and FLT3) occurred less frequently than what has been reported for other large AML patient cohorts that included younger patients [3, 21]. 

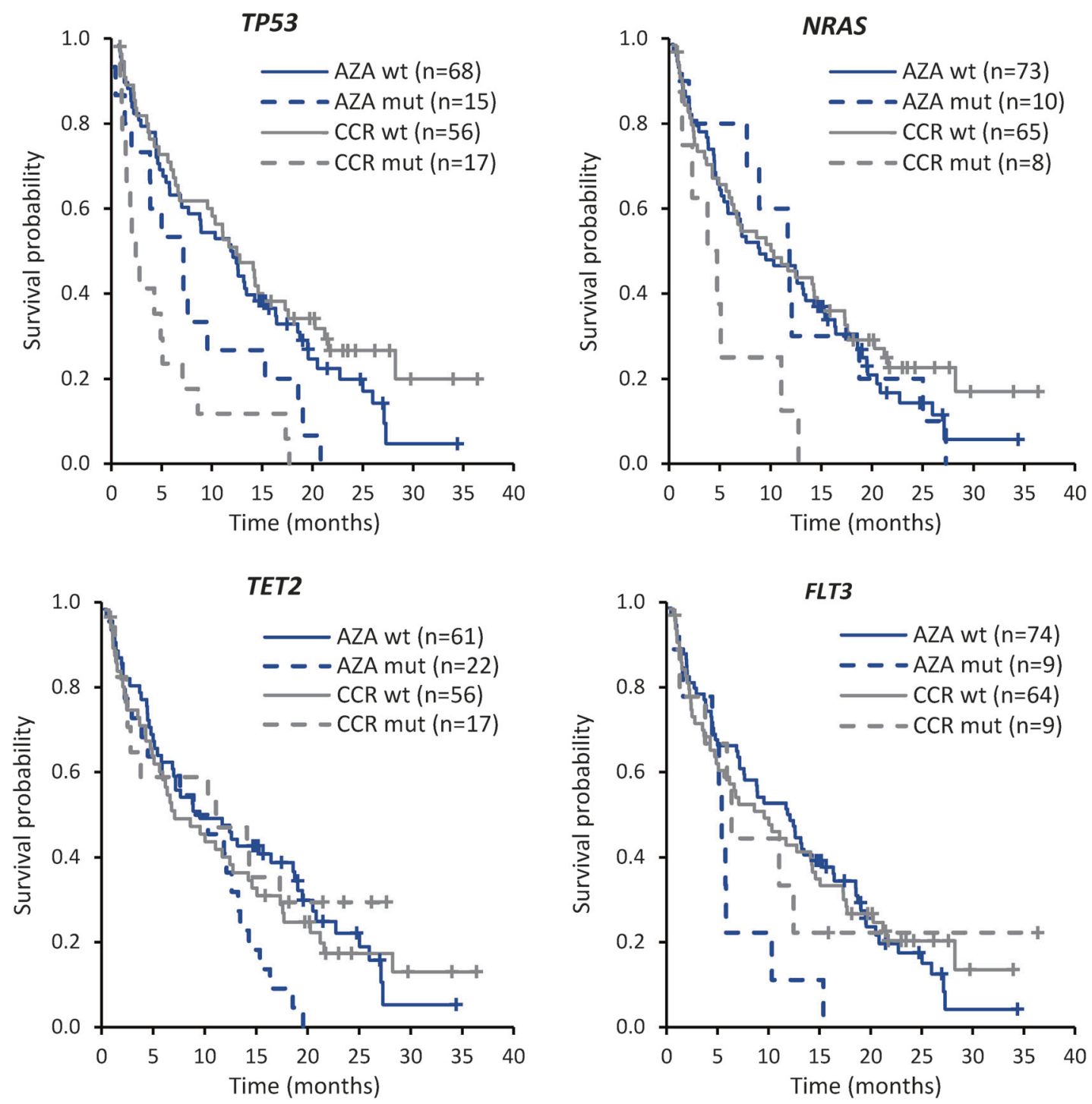

Fig. 5 Kaplan-Meier curves for gene mutations significantly $(P<0.05)$ associated with overall survival within treatment arms (mutant vs wildtype)

In this analysis, mutations in four genes were shown to significantly impact survival within treatment arms: mutant TP53 and NRAS in the CCR arm, and mutant FLT3 and TET2 in the azacitidine arm. Within the CCR arm, TP53 and NRAS mutations were associated with significantly reduced OS compared with patients with wild-type genes. TP53 mutations, which occur in $\sim 5-8 \%$ of all patients with AML [22, 23], are more frequently observed in older patients ( $21 \%$ of patients in the current study) and patients with abnormalities of chromosomes 5,7 , or $17 \mathrm{p}$, are associated with complex karyotype, and generally indicate a poor prognosis in hematologic malignancies regardless of treatment choice [3, 22, 24-29]. However, in keeping with the current study, it has been suggested that hypomethylating agents (HMAs) may be more effective than conventional care in patients with these mutations. In a study of decitabine treatment in patients with AML or MDS, those with TP53 mutations had a $100 \%$ response rate compared with a $41 \%$ response rate in patients with wild-type TP53 [30]. During decitabine treatment, TP53 VAF decreased rapidly to $<5 \%$ (though the mutation was never completely cleared); this was accompanied by bone marrow blast clearance in many instances. In the current analysis, median OS was prolonged by almost 5 months in patients with pretreatment TP53 mutations who received azacitidine compared with similar patients who received CCR. There was a significant correlation between higher TP53 VAF at baseline and decreased survival compared with patients with wild-type TP53 in both treatment arms, but the relative hazard was much greater in the CCR arm. The prognostic effects of NRAS mutations, which occur in $\sim 15 \%$ of patients with AML (12\% of patients in this study), typically at 
Table 2 Median OS between treatment arms (azacitidine vs CCR) for the most frequently mutated genes and genes involved in DNA methylation

\begin{tabular}{llll}
\hline Mutated gene & $\begin{array}{l}\text { AZA } \\
\text { Median OS, } \\
\text { months } \\
(95 \% \mathrm{CI})\end{array}$ & $\begin{array}{l}\text { CCR } \\
\text { Median OS, } \\
\text { months } \\
(95 \% \mathrm{CI})\end{array}$ & $\begin{array}{l}\text { Stratified } \\
P \text { Value }\end{array}$ \\
\hline TP53 & $7.2(3.9,18.6)$ & $2.4(1.5,7.1)$ & 0.093 \\
NRAS & $11.8(7.7, \mathrm{NR})$ & $4.3(2.3, \mathrm{NR})$ & 0.151 \\
FLT3 $^{\mathrm{a}}$ & $5.4(4.5, \mathrm{NR})$ & $6.4(3.8, \mathrm{NR})$ & 0.271 \\
TET2 & $9.6(4.5,13.5)$ & $11.1(2.8, \mathrm{NR})$ & 0.036 \\
IDH2 & $12.6(4.4, \mathrm{NR})$ & $12.5(5.6, \mathrm{NR})$ & 0.429 \\
DNMT3A & $12.6(7.0,20.8)$ & $10.3(3.8, \mathrm{NR})$ & 0.624 \\
RUNX1 & $13.5(8.8, \mathrm{NR})$ & $6.4(3.8, \mathrm{NR})$ & 0.496 \\
NPM1 & $7.3(4.5, \mathrm{NR})$ & $12.5(4.3,17.6)$ & 0.726 \\
ASXL1 & $18.7(4.8, \mathrm{NR})$ & $14.6(10.0, \mathrm{NR})$ & 0.643 \\
STAG2 & $19.5(11.9, \mathrm{NR})$ & $11.1(5.1, \mathrm{NR})$ & 0.722 \\
Any DNA & $11.1(5.8,15.4)$ & $12.5(4.3,17.6)$ & 0.248 \\
methylation gene & & & \\
\hline
\end{tabular}

${ }^{\mathrm{a}}$ FLT3-ITD and FLT3-TKD

b Includes IDH1, IDH2, DNMT3A, TET1, and TET2

$N R$ not reached

hotspot regions at codons 12,13 , and 61, are less clear. Their clinical implications may depend on the co-mutational context in which they occur, and patterns of NRAS comutations can vary by hotspots within genes [23, 31, 32]. For example, a recent study showed NPM1 mutations to be preferentially associated with $N R A S^{\mathrm{G} 12 / 13}$ but not with $N R A S^{\mathrm{Q} 61}$ and that OS outcomes were more favorable when $N R A S^{\mathrm{G} 12 / 13}$ mutations were accompanied by NPMI and DNMT3A mutations [3].

TET2 mutations occur in about $7-25 \%$ of patients with AML (25\% in this older patient population) [27, 33]. Although median OS within the azacitidine arm for patients with TET2 mutations differed by only 0.1 month compared with those without the mutation, the Kaplan-Meier curve separated after the estimated median, leading to a statistically significant difference in OS between the two groups. Median OS was $~ 1.5$ months longer in CCR-treated patients with TET2 mutations than in similar azacitidine-treated patients, which was unexpected based on a pathological feature associated with TET2 mutations (hypermethylation of DNA) and the purported activity of azacitidine (DNA demethylation) [34-36]. However, as there were a relatively small number of azacitidine-treated patients with TET2 mutations in this analysis $(n=22)$ this finding requires further confirmation in a larger patient population. When taken as a group, mutations in genes that regulate DNA methylation did not influence median OS with azacitidine or
CCR treatment. The prognostic consequences of mutations in FLT3 may vary based on co-occurring mutations; for example, when present with an NPM1 mutation in younger patients, prognosis is somewhat better than if accompanied by wild-type NPM1 [37]. Approximately $20 \%$ of AML patients present with FLT3 mutations although they are more common in younger patients with normal karyotype (only $12 \%$ of patients in the current study had an FLT3 mutation) [23, 38]. There was no statistical difference between azacitidine and CCR treatment in median OS of patients with FLT3 mutations, but within the azacitidine arm, presence of a FLT3 mutation at baseline was associated with poorer OS compared with wild-type FLT3.

Class-defining NPM1 and a provisional entity, RUNX1 [4], were among the most commonly mutated genes in the biomarker cohort. Although differences in survival were not statistically significant within treatment arms compared with the wild-type genes, mutations in NPMI appeared to confer somewhat poorer survival, in contrast to what has been shown in other AML cohorts [39, 40], in both the azacitidine and CCR treatment arms. Moreover, mutations in RUNX1 were associated with slightly better median OS in this analysis, although they have been associated with poorer prognosis in other studies [41, 42]. The number of patients in this analysis with mutant NPM1 $(n=25)$ or RUNX1 $(n=28)$ were relatively small, and these outcomes highlight a potential limitation of the data; namely, effects of isolated mutations or chromosomal defects provide only limited information by not considering cooperating pathogenic mechanisms at work in any given patient. Another limitation of this analysis is that changes in molecular and cytogenetic abnormalities during treatment were not captured.

The extraordinary heterogeneity and complexity of pathogenic mechanisms found in AML and the interplay among them in individual patients have made finding a cure-or even effective treatment-challenging. Nevertheless, increasing understanding of the genomic basis of AML and the introduction of new targeted therapies may allow the use of rational combination treatment regimens that include broadly effective agents such as azacitidine and an agent targeting a specific pathogenic pathway to improve patient outcomes. Studies in AML of azacitidine in combination with the BCL2 inhibitor, venetoclax (ClinicalTrials.gov NCT03466294), the mutant IDH inhibitors, enasidenib and ivosidenib (NCT02677922), and the mutant FLT3 inhibitors, gilteritinib (NCT02752035) and quizartinib (NCT01892371), are currently ongoing.

The data presented here suggest that azacitidine may be the preferred treatment for older patients with newly diagnosed AML with Adverse-risk cytogenetics who are not candidates for intensive chemotherapy, particularly those with chromosome 5, 7, and/or 17 abnormalities, and with 
complex or monosomal karyotypes. Moreover, older AML patients with TP53 or NRAS mutations may have prolonged survival when treated with azacitidine rather than with CCR. Outcomes of studies evaluating azacitidine as the backbone of combination regimens with targeted treatments are eagerly anticipated.

Acknowledgements The authors thank John Morrill and Jerry Weaver (Celgene Corporation) for assistance with data analysis. Editorial and administrative assistance was provided by Sheila Truten and Kelly Dittmore (Medical Communication Company, Inc.), sponsored by Celgene Corporation. This study was funded by Celgene Corporation.

Author contributions H. Döhner, S.S., C.L.B., J.F.S. and H. Dombret designed the study. H. Döhner, A.D., L.T., J.F.S., M.D.M., R.M.S., T.B.d-C., H.K.A-.A., V.S., P.V., C.L.B., K.J.M., B.S.S, S.S., L.B. and H. Dombret performed the research and analyzed the data. A.D, L.T. and K.J.M. performed molecular analyses. N.T. performed statistical analyses. All authors contributed to, revised, and approved manuscript content. All authors gave approval for submission to the journal and are fully responsible for all content and editorial decisions.

\section{Compliance with ethical standards}

Conflict of interest H. Döhner: Advisory Boards (with honoraria): AbbVie, Agios, Amgen, Astellas, Astex Pharmaceuticals, Celator, Celgene Corporation, Janssen, Jazz Pharmaceuticals, Novartis, Seattle Genetics, Sunesis. J.F.S.: Advisory Committees, AbbVie, Celgene Corporation, Genentech, Gilead, Janssen, Roche, Takeda; Consultancy, AbbVie, Celgene Corporation, Genentech, Gilead, Janssen, Roche, Takeda; Honoraria, AbbVie, Celgene Corporation, Genentech, Gilead, Janssen, Roche, Takeda; Travel support, AbbVie, Celgene Corporation; Research Funding, AbbVie, Janssen; Speakers Bureau, AbbVie, Celgene Corporation, Gilead, Janssen, Roche. R.M.S.: Consultancy, AbbVie, Agios, Amgen, BristolMeyersSquibb, Celator, Celgene Corporation, Janssen, Juno Therapeutics, Karyopharm, Merck, Novartis, Roche/Genentech, Pfizer, Seattle Genetics, Sunesis Pharmaceuticals, Xenetic Biosciences; Research Funding, Agios, Celator, Karyopharm, Novartis, Pfizer; Advisory Committees, Celgene Corporation. H.K.A.-A.: Consultancy, Honoraria and Research Funding, Celgene; Consultancy, Honoraria and Research Funding, Novartis. V.S.: Consultancy, Amgen, Astex, Celgene Corporation, Janssen, Novartis, Onconova; Honoraria, Celgene Corporation, Janssen, Novartis; Research Funding, Celgene Corporation. P.V.: Honoraria and Research Funding, Celgene Corporation. S.S., C.L.B., L.T., K.J.M., B.S.S.: Employment and equity ownership, Celgene Corporation. L.B.: Advisory Committees BristolMeyersSquibb, Boehringer Ingelheim, Celgene Corporation, Jazz Pharmaceuticals, Novartis, MSD Sharp \& Dohme GmbH, Merck, Seattle Genetics. The remaining authors declare that they have no conflict of interest.

Open Access This article is licensed under a Creative Commons Attribution 4.0 International License, which permits use, sharing, adaptation, distribution and reproduction in any medium or format, as long as you give appropriate credit to the original author(s) and the source, provide a link to the Creative Commons license, and indicate if changes were made. The images or other third party material in this article are included in the article's Creative Commons license, unless indicated otherwise in a credit line to the material. If material is not included in the article's Creative Commons license and your intended use is not permitted by statutory regulation or exceeds the permitted use, you will need to obtain permission directly from the copyright holder. To view a copy of this license, visit http://creativecommons. org/licenses/by/4.0/.

\section{References}

1. Dohner H, Estey EH, Amadori S, Appelbaum FR, Buchner T, Burnett AK, et al. Diagnosis and management of acute myeloid leukemia in adults: recommendations from an international expert panel, on behalf of the European LeukemiaNet. Blood. 2010;115:453-74.

2. Bullinger L, Dohner K, Dohner H. Genomics in acute myeloid leukemia diagnosis and pathways. J Clin Oncol. 2017;35:1-13.

3. Papaemmanuil E, Gerstung M, Bullinger L, Gaidzik VI, Paschka $\mathrm{P}$, Roberts ND, et al. Genomic classification and prognosis in acute myeloid leukemia. N Engl J Med. 2016;374:2209-21.

4. Dohner H, Estey E, Grimwade D, Amadori S, Appelbaum FR, Buchner T, et al. Diagnosis and management of AML in adults: 2017 ELN recommendations from an international expert panel. Blood. 2017;129:424-47.

5. Grimwade D, Walker H, Oliver F, Wheatley K, Harrison C, Harrison G, et al. The importance of diagnostic cytogenetics on outcome in AML: analysis of 1612 patients entered into the MRC AML 10 trial. The medical research council adult and children's leukaemia working parties. Blood. 1998;92:2322-33.

6. Patel JP, Gonen M, Figueroa ME, Fernandez H, Sun Z, Racevskis $\mathrm{J}$, et al. Prognostic relevance of integrated genetic profiling in acute myeloid leukemia. N Engl J Med. 2012;366:1079-89.

7. Arber DA, Orazi A, Hasserjian R, Thiele J, Borowitz MJ, Le Beau $\mathrm{MM}$, et al. The 2016 revision to the World Health Organization classification of myeloid neoplasms and acute leukemia. Blood. 2016;127:2391-405.

8. Dombret H, Seymour JF, Butrym A, Wierzbowska A, Selleslag D, Jang $\mathrm{JH}$, et al. International phase 3 study of azacitidine vs conventional care regimens in older patients with newly diagnosed AML with $>30 \%$ blasts. Blood. 2015;126:291-9.

9. National comprehensive cancer network $(\mathrm{NCCN})$ clinical practice guidelines in oncology ${ }^{\mathrm{TM}}$. Acute Myeloid Leukemia v1.2009. https://www.nccn.org/professionals/physician_gls/default.aspx

10. Gandrud C. simPH: an R package for illustrating estimates from Cox proportional hazard models including for interactive and nonlinear effects. J Stat Softw. 2015;65:1-20.

11. Lazarevic V, Horstedt AS, Johansson B, Antunovic P, Billstrom $\mathrm{R}$, Derolf $\mathrm{A}$, et al. Incidence and prognostic significance of karyotypic subgroups in older patients with acute myeloid leukemia: the Swedish population-based experience. Blood Cancer J. 2014;4:e188.

12. Marchesi F, Annibali O, Cerchiara E, Tirindelli MC, Avvisati G. Cytogenetic abnormalities in adult non-promyelocytic acute myeloid leukemia: a concise review. Crit Rev Oncol Hematol. 2011;80:331-46.

13. Nazha A, Kantarjian HM, Bhatt VR, Nogueras-Gonzalez G, Cortes JE, Kadia T, et al. Prognostic implications of chromosome 17 abnormalities in the context of monosomal karyotype in patients with acute myeloid leukemia and complex cytogenetics. Clin Lymphoma Myeloma Leuk. 2014;14:163-71.

14. Seymour JF, Dohner H, Butrym A, Wierzbowska A, Selleslag D, Jang JH, et al. Azacitidine improves clinical outcomes in older patients with acute myeloid leukaemia with myelodysplasiarelated changes compared with conventional care regimens. BMC Cancer. 2017;17:852.

15. Ravandi F, Issa JP, Garcia-Manero G, O’Brien S, Pierce S, Shan J, et al. Superior outcome with hypomethylating therapy in patients with acute myeloid leukemia and high-risk myelodysplastic syndrome and chromosome 5 and 7 abnormalities. Cancer. 2009; 115:5746-51. 
16. Vardiman JW, Thiele J, Arber DA, Brunning RD, Borowitz MJ, Porwit A, et al. The2008 revision of the World Health Organization (WHO) classification of myeloid neoplasms and acute leukemia: rationale and important changes. Blood. 2009;114:93751 .

17. Dicker F, Haferlach C, Sundermann J, Wendland N, Weiss T, Kern W, et al. Mutation analysis for RUNX1, MLL-PTD, FLT3ITD, NPM1 and NRAS in 269 patients with MDS or secondary AML. Leukemia. 2010;24:1528-32.

18. Shen Y, Zhu YM, Fan X, Shi JY, Wang QR, Yan XJ, et al. Gene mutation patterns and their prognostic impact in a cohort of 1185 patients with acute myeloid leukemia. Blood. 2011;118:5593603.

19. Richardson RB. p53 mutations associated with aging-related rise in cancer incidence rates. Cell Cycle. 2013;12:2468-78.

20. Tsai CH, Hou HA, Tang JL, Liu CY, Lin CC, Chou WC, et al. Genetic alterations and their clinical implications in older patients with acute myeloid leukemia. Leukemia. 2016;30:1485-92.

21. Cancer Genome Atlas Research N. Genomic and epigenomic landscapes of adult de novo acute myeloid leukemia. N Engl J Med. 2013;368:2059-74.

22. Stengel A, Kern W, Haferlach T, Meggendorfer M, Fasan A, Haferlach C. The impact of TP53 mutations and TP53 deletions on survival varies between AML, ALL, MDS and CLL: an analysis of 3307 cases. Leukemia. 2017;31:705-11.

23. Dohner H, Weisdorf DJ, Bloomfield CD. Acute myeloid leukemia. N Engl J Med. 2015;373:1136-52.

24. Devillier R, Mansat-De Mas V, Gelsi-Boyer V, Demur C, Murati A, Corre J, et al. Role of ASXL1 and TP53 mutations in the molecular classification and prognosis of acute myeloid leukemias with myelodysplasia-related changes. Oncotarget. 2015;6:838896.

25. Stirewalt DL, Kopecky KJ, Meshinchi S, Appelbaum FR, Slovak ML, Willman CL, et al. FLT3, RAS, and TP53 mutations in elderly patients with acute myeloid leukemia. Blood. 2001;97: 3589-95.

26. Jung SH, Kim YJ, Yim SH, Kim HJ, Kwon YR, Hur EH, et al. Somatic mutations predict outcomes of hypomethylating therapy in patients with myelodysplastic syndrome. Oncotarget. 2016; 7:55264-75.

27. Ohgami RS, Ma L, Merker JD, Gotlib JR, Schrijver I, Zehnder JL, et al. Next-generation sequencing of acute myeloid leukemia identifies the significance of TP53, U2AF1, ASXL1, and TET2 mutations. Mod Pathol. 2015;28:706-14.

28. Kadia TM, Jain P, Ravandi F, Garcia-Manero G, Andreef M, Takahashi K, et al. TP53 mutations in newly diagnosed acute myeloid leukemia: clinicomolecular characteristics, response to therapy, and outcomes. Cancer. 2016;122:3484-91.

29. Rucker FG, Schlenk RF, Bullinger L, Kayser S, Teleanu V, Kett $\mathrm{H}$, et al. TP53 alterations in acute myeloid leukemia with complex karyotype correlate with specific copy number alterations, monosomal karyotype, and dismal outcome. Blood. 2012;119:2114-21.
30. Welch JS, Petti AA, Miller CA, Fronick CC, O'Laughlin M, Fulton RS, et al. TP53 and decitabine in acute myeloid leukemia and myelodysplastic syndromes. N Engl J Med. 2016;375:202336.

31. Bacher U, Haferlach T, Schoch C, Kern W, Schnittger S. Implications of NRAS mutations in AML: a study of 2502 patients. Blood. 2006;107:3847-53.

32. Kunimoto H, Meydan C, Nazir A, Whitfield J, Shank K, Rapaport F, et al. Cooperative epigenetic remodeling by TET2 loss and NRAS mutation drives myeloid transformation and MEK inhibitor sensitivity. Cancer Cell. 2018;33:44-59 e8.

33. Bejar R, Lord A, Stevenson K, Bar-Natan M, Perez-Ladaga A, Zaneveld $\mathrm{J}$, et al. TET2 mutations predict response to hypomethylating agents in myelodysplastic syndrome patients. Blood. 2014;124:2705-12.

34. Figueroa ME, Abdel-Wahab O, Lu C, Ward PS, Patel J, Shih A, et al. Leukemic IDH1 and IDH2 mutations result in a hypermethylation phenotype, disrupt TET2 function, and impair hematopoietic differentiation. Cancer Cell. 2010;18:553-67.

35. Hollenbach PW, Nguyen AN, Brady H, Williams M, Ning Y, Richard $\mathrm{N}$, et al. A comparison of azacitidine and decitabine activities in acute myeloid leukemia cell lines. PLoS ONE. 2010;5:e9001.

36. Pleyer L, Greil R. Digging deep into "dirty" drugs-modulation of the methylation machinery. Drug Metab Rev. 2015;47:252-79.

37. Gale RE, Green C, Allen C, Mead AJ, Burnett AK, Hills RK, et al. The impact of FLT3 internal tandem duplication mutant level, number, size, and interaction with NPM1 mutations in a large cohort of young adult patients with acute myeloid leukemia. Blood. 2008;111:2776-84.

38. Grimwade D, Ivey A, Huntly BJ. Molecular landscape of acute myeloid leukemia in younger adults and its clinical relevance. Blood. 2016;127:29-41.

39. Dohner K, Schlenk RF, Habdank M, Scholl C, Rucker FG, Corbacioglu A, et al. Mutant nucleophosmin (NPM1) predicts favorable prognosis in younger adults with acute myeloid leukemia and normal cytogenetics: interaction with other gene mutations. Blood. 2005;106:3740-6.

40. Verhaak RG, Goudswaard CS, van Putten W, Bijl MA, Sanders MA, Hugens W, et al. Mutations in nucleophosmin (NPM1) in acute myeloid leukemia (AML): association with other gene abnormalities and previously established gene expression signatures and their favorable prognostic significance. Blood. 2005; 106:3747-54.

41. Schnittger S, Dicker F, Kern W, Wendland N, Sundermann J, Alpermann T, et al. RUNX1 mutations are frequent in de novo AML with noncomplex karyotype and confer an unfavorable prognosis. Blood. 2011;117:2348-57.

42. Gaidzik VI, Teleanu V, Papaemmanuil E, Weber D, Paschka P, Hahn J, et al. RUNX1 mutations in acute myeloid leukemia are associated with distinct clinico-pathologic and genetic features. Leukemia. 2016;30:2160-8. 